\title{
PICOSECOND TRANSIENT ABSORPTION SPECTRA AND THE TWISTED INTRAMOLECULAR CHARGE TRANSFER PHENOMENON
}

\author{
J. Dobkowski, Z.R. Grabowski*, J. JaSNY aND Z. ZIELIŃSkI \\ Institute of Physical Chemistry, Polish Academy of Sciences \\ Kasprzaka 44, 01-224 Warszawa, Poland
}

(Received April 24, 1995)

\section{Dedicated to Professors Zdzisław Ruziewicz and Krzysztof Pigon' on the occasion of their 70th birthdays ${ }^{\dagger}$}

An improved model of a computer-controlled picosecond absorption spectrometer was designed and constructed. Its operation is described, and applied to the measurement of transient spectra of $p-N, N$-dioctylamino-acetophenone. The spectroscopy and photophysics of this compound are compared to the other compounds exhibiting the dual fluorescence, assigned usually to the adiabatic formation of the "twisted" intramolecular charge transfer excited state. The data are discussed in relation to alternative suggestion of an inversion of the pyramidal amino nitrogen atom. The whole evidence supports the decoupling of the $\pi$-electronic systems of $D^{+}$and $A^{-}$in the $D^{+}-A^{-}$polar excited state, in full accord with the "twisted" intramolecular charge transfer model. The peculiar behaviour of the long alkyl $-\mathrm{NR}_{2}$ derivatives (favouring the "twisted" intramolecular charge transfer state emission) seems to be primarily due to the decreasing oxidation potential of such electron donor groups.

PACS numbers: $36.20 . \mathrm{Kd}, 06.60$. In

\section{Introduction}

Numerous molecules built of an electron donor $(D)$ and electron acceptor $(A)$ linked together by the single bond (i.e., $D-A$ molecules) exhibit a peculiar behaviour in their low-laying electronically excited states. A polar solvent assisted, very fast but thermally activated adiabatic reaction occurs, leading to a highly polar excited state. In favourable cases (if there is in the ground state a considerable barrier to internal rotation) two fluorescence bands appear, the primary

*and the College of Science in Warsaw.

†Submitted on invitation of the Institute of Physical and Theoretical Chemistry, Technical University of Wrocław, Wrocław, Poland. 
emission described as $F_{\mathrm{b}}$, the secondary (from the polar emitting state) $-F_{\mathrm{a}}$. The nature of the polar emitting state, since the discovery of the dual fluorescence of $p-N, N$-dimethylamino-benzonitrile (DMABN) by Lippert et al. [1] had been the subject of numerous controversial hypotheses. Now, the most generally accepted hypothesis [2] is the one of the "twisted" intramolecular charge transfer state (TICT) according to which the $D$ and $A$ moieties, limited in their structural degrees of flexibility, twist to orthogonality of their $\pi$-electronic systems $[3,4]$. Among the recently supported principally opposing hypotheses there are those assigning the dual fluorescence of DMABN and structurally related compounds either to the formation of exciplexes with the solvent [5], or to the pseudo Jahn-Teller distortion [6,7]. Marginally: both these views mutually oppose.
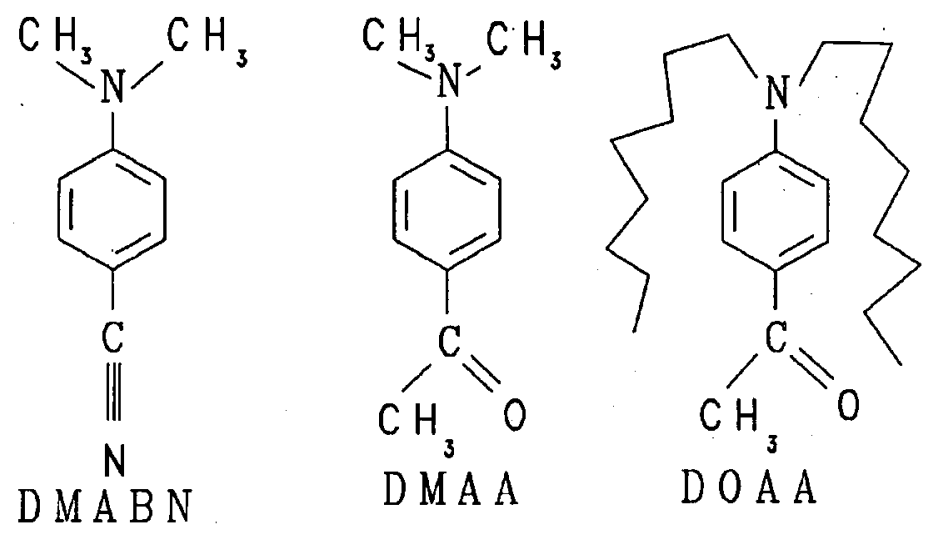

Cognition of the molecular structure of an excited singlet state is most frequently done by one of the following ways: (i) quantum chemical calculation at different levels of sophistication; (ii) comparison of the electronic absorption (from the ground state, $\left.S_{n} \leftarrow S_{0}\right)$ and fluorescence spectra $\left(S_{1} \rightarrow S_{0}\right)$ (Franck-Condon analysis; Stokes' shift; solvatochromic shifts; polarization; factors affecting the kinetics); (iii) assignment of the transient absorption spectrum from the excited state, $S_{n} \leftarrow S_{1}$.

Quantum chemical calculations, starting from early papers [3] to the recent highly advanced quantum-chemical description of the DMABN molecule by Roos et al. [8], support the existence of a highly polar state $(\mu \approx 15-19 \mathrm{D})$ as onc of the lowest excited states in the free molecule in its twisted (orthogonal) conformation; stabilization by polar solvents turns this state to the lowest excited singlet state (inaccessible directly in absorption, due to the Franck-Condon rule). Such result has been obtained also for $p$-dimethylamino-benzaldehyde (DMABA), a carbonyl derivative closely related to, and of the same $\pi$-electronic structure as, DMAA and DOAA [9].

Luminescence was the main evidence in large majority of the relevant papers. For DMAA the evidence is first delivered by the comparative study of the model compounds [10]. 
Transient absorption spectra, in a few cases reported until now, give the real key to understand the electronic structure of the $F_{\mathrm{a}}$ emitter. For DMABN [11] and DMAA [12] the transient absorption spectra in the visible were matching the spectra of the corresponding radical anion, $A^{-}$: that of benzonitrile ${ }^{-}$or acetophenone ${ }^{-}$, respectively [13].

Considering the transient absorption spectra the most important evidence in disclosing the nature of the discussed polar state, we decided to build the picosecond absorption spectrometer. The inspiration was borrowed initially from the apparatus built in Bordeaux by Rullière and his students [14], then modified and completed in many respects.

Zachariasse et al. [6] reported that the dialkylamino-benzonitriles with the methyl groups replaced by longer alkyls emit the long-wave $F_{a}$ band with much higher relative yield than DMABN. For the internal rotation one could expect the contrary: large, voluminous groups should slow down the internal rotation. The effect of the large dialkylamino-groups awaited an explanation.

The subject of this paper is a comparison of the spectra and photophysics of DMAA and DOAA, specially of the transient absorption spectra, to elucidate the nature of the highly polar (TICT ?) states. The measurements of the transient absorption were carried out with the just built original picosecond absorption spectrometer.

\section{Experimental}

\subsection{Compounds and synthesis}

Synthesis of 4- $N, N$-dioctylamino-acetophenone (DOAA): 4-amino acetophenone was boiled for 12 hours in $n$-octanol with fourfold excess of $n$-octyl iodide, fivefold molar quantity of anhydrous $\mathrm{K}_{2} \mathrm{CO}_{3}$ and a catalytic quantity of $\mathrm{KI}$. Excess of octyl iodide and octanol was removed by distillation with water vapour, the cooled residual extracted with ether, dried, and after removal of ether dissolved in hexane. After filtering off some crystalline 4-octylamino-acetophenone, the solution was evaporated and the residual distilled. Pale yellowish liquid, b.p.: $278^{\circ} \mathrm{C}$ $(p=10 \mathrm{~mm} \mathrm{Hg}) ;{ }^{1} \mathrm{H} \mathrm{NMR}\left(\mathrm{CDCl}_{3}\right)$ supported the structure.

DMAA was purified as described earlier [9]. Ether, tetrahydrofuran (THF) and acetonitrile (ACN) were of spectroscopic purity (MERCK).

\subsection{Stationary fluorescence measurements}

Stationary fluorescence spectra were measured with Jasny spectrofluorimeter [15], much modified and equipped with a photon counting detection system. The spectra are corrected for the instrumental response.

\section{Picosecond spectrometer}

\subsection{Construction and operation}

The computer-controlled picosecond spectrometer (Fig. 1) is based on the passively mode-locked Nd:YAG laser system (EKSPLA, Lithuania) as the light source. The negative feedback control system stabilizes the mean pulse energy. A single pulse $(\tau \approx 25 \mathrm{ps}$ fwhm, $\lambda=1064 \mathrm{~nm})$ is selected by an opto-electronic 


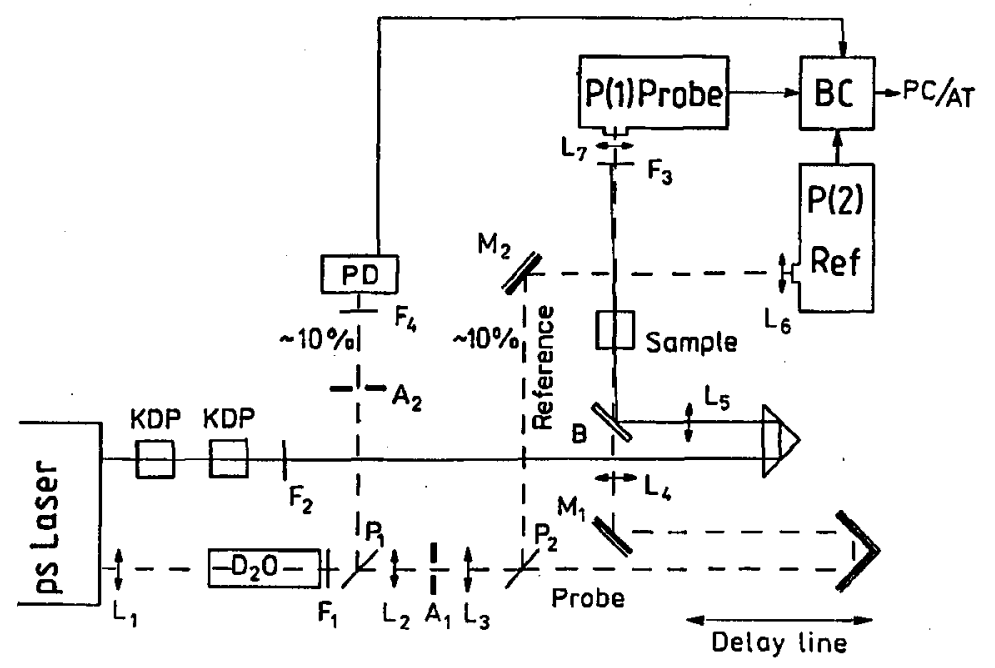

Fig. 1. Optical scheme of the picosecond spectrometer for transient absorption measurements. $\mathrm{L}_{1}$ - lens, $f=650 \mathrm{~mm} ; \mathrm{F}_{1}, \mathrm{~F}_{2}$ - optical edge filters (cut-off $1064 \mathrm{~nm}$ ); $\mathrm{P}_{1}$, $\mathrm{P}_{2}$ - quartz plates; $\mathrm{L}_{2}, \mathrm{~L}_{3}$ - achromatic lens, $f=85 \mathrm{~mm} ; \mathrm{A}_{1}$ - aperture $0.2-0.6 \mathrm{~mm}$; $\mathrm{M}_{1}, \mathrm{M}_{2}$ - mirrors; $\mathrm{L}_{4}-$ achromatic lens, $f=192 \mathrm{~mm}$; $\mathrm{L}_{5}-$ lens, $f=200 \mathrm{~mm}$; B dielectric mirror (chromatic beam splitter) reflective in UV, transparent in the visible; $\mathrm{F}_{3}$ - optical filter (cut-off $\mathrm{UV}$ ); $\mathrm{L}_{6}, \mathrm{~L}_{7}$ - achromatic collective lens, $f=35 \mathrm{~mm} ; \mathrm{P}(1)$, $\mathrm{P}(2)$ - polychromators with photodiode array detectors; $\mathrm{BC}$ - buffer memory and contol unit; $\mathrm{A}_{2}-$ controlled aperture; $\mathrm{F}_{4}-$ interference filter, $\lambda_{\max }=600 \mathrm{~nm} ; \mathrm{PD}-$ photodiode.

pulse selector, amplified, and split in two beams (pulse energy about $20 \mathrm{~mJ}$ each). The first beam is directed into the KDP crystals to generate higher harmonic frequencies. Third harmonic, $\lambda=355 \mathrm{~nm}$, is used as the excitation beam.

The other beam of the fundamental frequency is focused inside the long cell with $\mathrm{D}_{2} \mathrm{O}$ to generate the pulse of spectral continuum extending between 430 and $800 \mathrm{~nm}$ (cf. Fig. 3A, curve a), used as the probe. As any large variation of the pulse intensity can affect the spectral distribution of the continuum [16], the intensity of the latter is controlled by a photodiode (PD) at the fixed $\lambda=600 \mathrm{~nm}$.

The spectral distribution is monitored by splitting the continuum in two, the reference and the probe beam, each focused into the slit of the corresponding polychromator, $\mathrm{P}(2)$ or $\mathrm{P}(1)$, respectively. Each Jasny model polychromator contains the concave holographic grating (JOBIN-YVON) with a flat focusing field where the photodiode array (RETICON RL 1024 S) is situated. Relative aperture $f / 3.2$, spectral resolution $\approx 0.4 \mathrm{~nm} /$ diode.

The analog signals from the diode arrays are digitized and transmitted to the buffer memory, and next to the PC. The probe pulses are delayed in the specially designed variable optical delay line with the roof mirrors (active area $\phi=18 \mathrm{~mm}$ each). The length of the optical path in the loop (maximum delay $3.4 \mathrm{~ns}$, single step $\Delta t=0.1 \mathrm{ps}$ ) can be set manually or preset with a computer-controlled step motor. 
Both, probe and excitation beam, cross nearly collinearly the sample (Fig. 1). Finally the probe beam is focused in the slit of the probe polychromator $\mathrm{P}(1)$, spectrally resolved and detected by the photodiode array, as in the case of the reference channel.

During the experiments with multiple excitation laser pulses, there is a danger of accumulation of any stable photoproduct in the optical path in the sample, specially in viscous or rigid media. Therefore, a mechanical device is constructed which, before every new excitation pulse, moves the sample in the plane perpendicular to the optical path in such way that the focus of the laser beam draws a Lissajoux curve, and each excitation pulse irradiates a new, intact part of the sample volume [17].

\subsection{Measurement procedure}

First, the intensities of 50 pulses of the picosecond continuum are measured by the PD photodiode, and their mean $(\bar{I})$ and standard deviation $(\sigma)$ are statistically evaluated by the computer program. Only the laser shots resulting in continuum intensities within the range $\bar{I} \pm \sigma$ are processed (Fig. 2).

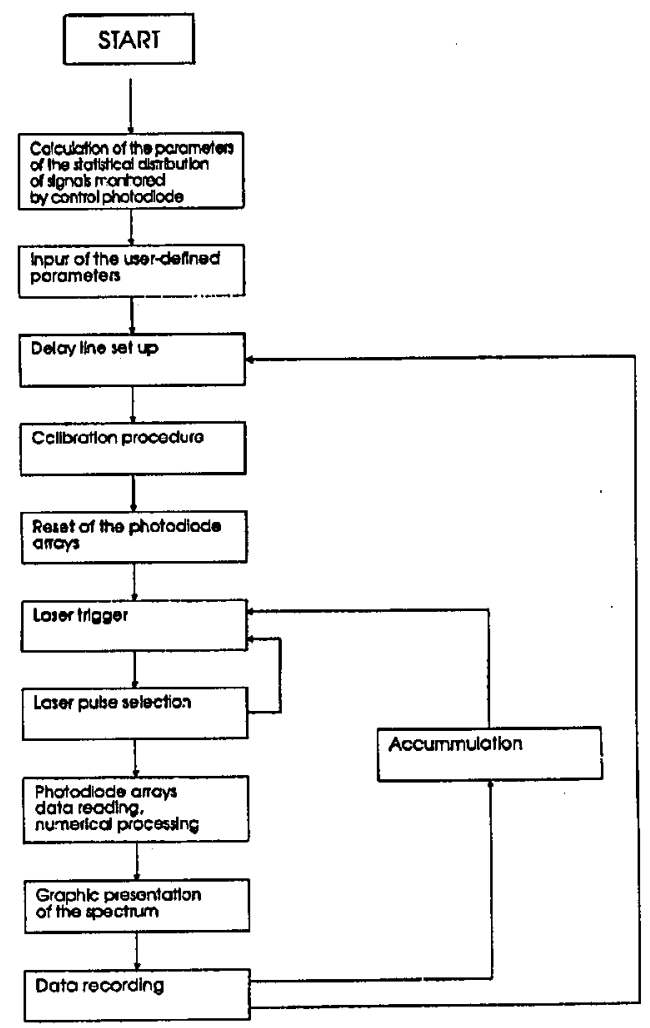

Fig. 2. Operational scheme of the picosecond spectrometer. 
The transient absorption spectrum at a given delay time $t_{\mathrm{d}}$ is calculated from the probe and reference beam signals $\left(S_{\mathrm{P}}\right.$ and $S_{\mathrm{R}}$, respectively), with $(S)$ and without $\left(S^{0}\right)$ excitation. The transient absorbance is

$$
A\left(\lambda, t_{\mathrm{d}}\right)=\log \left[\frac{S_{\mathrm{R}}\left(\lambda, t_{\mathrm{d}}\right)}{S_{\mathrm{P}}\left(\lambda, t_{\mathrm{d}}\right)} K(\lambda)\right],
$$

where the calibration curve

$$
K(\lambda) \equiv S_{\mathrm{P}}^{0}(\lambda) / S_{\mathbf{R}}^{0}(\lambda)
$$

The calibration was carried out for each series of experiments. To improve the $S / N$ ratio, $25-100$ spectra are accumulated. The spectra corresponding to Eqs. (1) and (2) are exemplified in Fig. 3.

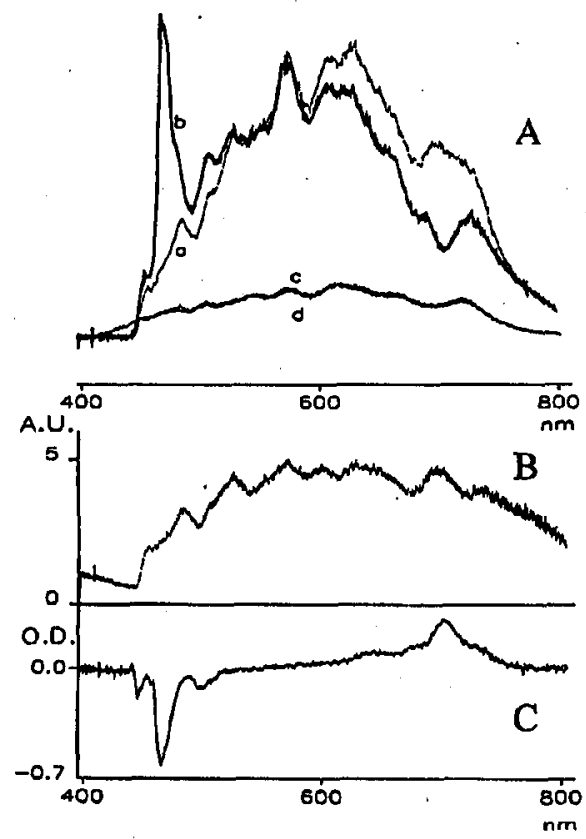

Fig. 3. Transient absorption of pyrene in cyclohexane, delay time $t_{\mathrm{d}}=100 \mathrm{ps}$. Top: ps-continuum as detected by the probe array (P(1) in Fig. 1), a - without, $S_{\mathrm{P}}^{0}(\lambda)$, and $\mathrm{b}$ - with the excitation pulse, $S_{\mathrm{P}}(\lambda) ; \mathrm{c}, \mathrm{d}$ - the same detected by the reference array $\left(\mathrm{P}(2)\right.$ in Fig. 1), $S_{\mathrm{R}}^{0}(\lambda)$ and $S_{\mathrm{R}}(\lambda)$, respectively. Middle part: calibration curve, $K(\lambda)$, as defined in Eq. (2). Bottom: transient spectrum, according to Eq. (1); transient absorption in the long-wave range, peaking around $700 \mathrm{~nm}$, and stimulated emission (negative absorption) between 440 and $513 \mathrm{~nm}$.

\section{Results}

\subsection{Stationary absorption and fluorescence}

In liquid solvents DOAA emits two weak fluorescence bands, the "normal" fluorescence $F_{\mathrm{b}}$, and the "anomalous" fluorescence $F_{\mathrm{a}}$ (Fig. 4). Their quantum 


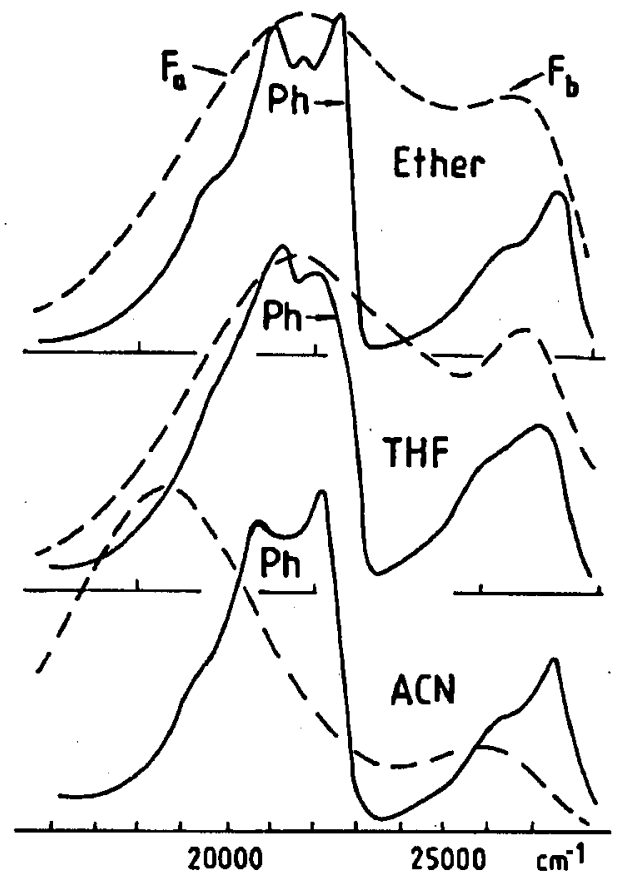

Fig. 4. Luminescence spectra of DOAA in three solvents, excitation $30000 \mathrm{~cm}^{-1}$ : solid curves $-T=77 \mathrm{~K}$, fluorescence and phosphorescence (Ph); dashed curves $-T=$ $293 \mathrm{~K}$, two fluorescence bands, "normal" $F_{\mathrm{b}}$, and "anomalous" or "TICT" emission $F_{\mathrm{a}}$.

\section{TABLE}

Comparison of the two fluorescences, $F_{\mathrm{b}}$ and $F_{\mathrm{a}}$, of DMAA and DOAA, $T=293 \mathrm{~K}$ : fluorescence maxima $\left(\nu_{f}\right)$ and quantum yields $\left(\phi_{f}\right)$.

\begin{tabular}{c|c|c|c|c|r}
\hline \hline \multicolumn{2}{c|}{} & \multicolumn{2}{c|}{ DMAA } & \multicolumn{2}{c}{ DOAA } \\
\hline Solvent & Band & $\nu_{f}\left[\mathrm{~cm}^{-1}\right]$ & $\phi_{f}$ & $\nu_{f}\left[\mathrm{~cm}^{-1}\right]$ & \multicolumn{1}{c}{$\phi_{f}$} \\
\hline Ether & $\mathrm{F}_{b}$ & $\ldots$ & $\ldots$ & 27100 & $4 \times 10^{-4}$ \\
& $\mathrm{~F}_{a}$ & $\ldots$ & $\ldots$ & 21500 & $1.3 \times 10^{-3}$ \\
\hline THF & $\mathrm{F}_{b}$ & $\ldots$ & $\ldots$ & 27100 & $4 \times 10^{-4}$ \\
& $\mathrm{~F}_{a}$ & $\ldots$ & $\ldots$ & 21700 & $1.3 \times 10^{-3}$ \\
\hline ACN & $\mathrm{F}_{b}$ & 26300 & 0.0018 & 25900 & 0.0025 \\
& $\mathrm{~F}_{a}$ & 17900 & 0.008 & 18600 & 0.016 \\
\hline
\end{tabular}

$\phi_{f}$ values of DMAA in ACN: from Ref. [18].

yields strongly depend on the solvent polarity (Table). At $77 \mathrm{~K}$ only the $F_{\mathrm{b}}$ fluorescence is observed, along with an intense phosphorescence (Ph) (Fig. 4). In ACN solutions of DOAA the $F_{\mathrm{a}} / F_{\mathrm{b}}$ ratio reaches its maximum at $T \approx 315 \mathrm{~K}$. 


\subsection{Fluorescence kinetics}

Preliminary experiments demonstrated that the DOAA fluorescence in ACN exhibits a typical bimodal kinetics: the $F_{\mathrm{b}}$ band measured at $\lambda=360 \mathrm{~nm}$ has a fast decay, $T \leq 20 \mathrm{ps} ; F_{\mathrm{a}}$, monitored at $\lambda=580 \mathrm{~nm}$, has a corresponding rise; both are followed by a slow common decay of the equilibrated system, $B^{*} \rightleftharpoons A^{*}$. Such behaviour is very similar to the parent-daughter relation observed in the photophysical kinetics of DMAA $[18,19]$.

\subsection{Transient absorption spectra}

Transient absorption spectra of DOAA were recorded in several aprotic solvents of different polarity. Examples are shown in Fig. 5. In weakly polar solvents the absorption maximum is observed at $\lambda \approx 463 \mathrm{~nm}$. After $2 \mathrm{~ns}$ this maximum does not change, whereas the shoulder at $\lambda \approx 500 \mathrm{~nm}$ disappears.

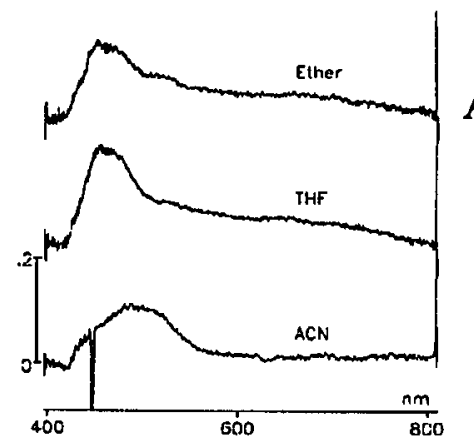

A
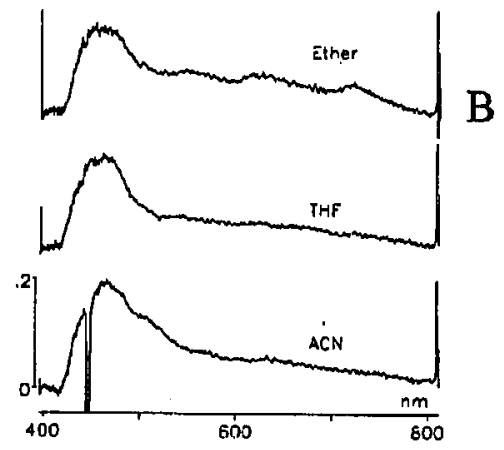

Fig. 5. Transient absorption spectra of DOAA in three solvents of different polarity, $T=293 \mathrm{~K} . \mathrm{A}-$ delay time $t_{\mathrm{d}}=100 \mathrm{ps} ; \mathrm{B}-t_{\mathrm{d}}=2000 \mathrm{ps}$. Optical density scale indicated at the ordinate axis.

In ACN at short $t_{\mathrm{d}}$ the absorption band has a broad maximum at $\lambda \approx$ 480-506 nm. At longer $t_{\mathrm{d}}$ the latter absorption decays, giving rise to the band at $\lambda \approx 465 \mathrm{~nm}$, similar to those in ether or THF. The temporal evolution of the absorption spectrum of DOAA in ACN is shown in Fig. 6. 


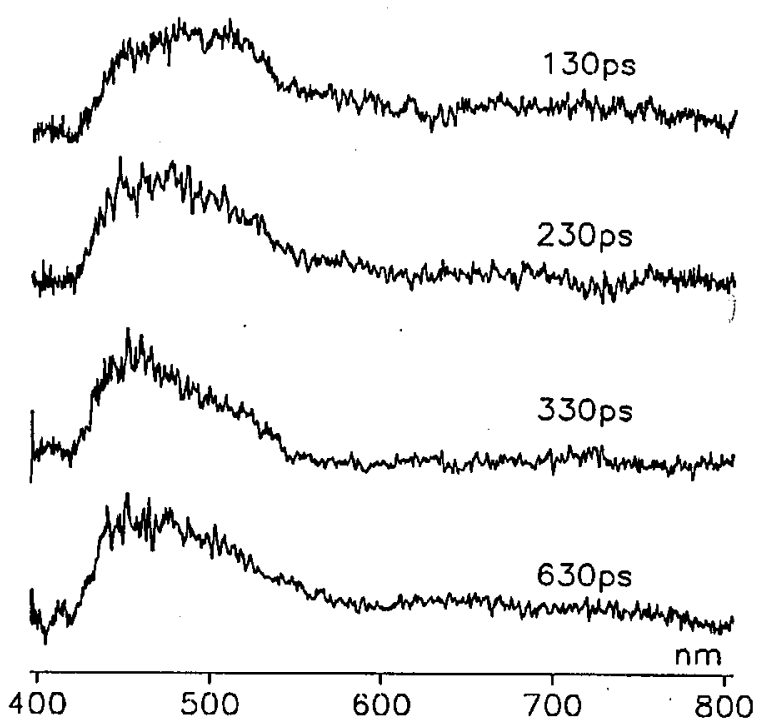

Fig. 6. Temporal evolution of the transient absorption spectrum of DOAA in ACN, $T=293 \mathrm{~K}$. Delay time $t_{\mathrm{d}}$ is indicated.

\section{Discussion}

\subsection{Assignment of the transienl absorption bands of DOAA}

The transient absorption spectra of DOAA (Figs. 5, 6) reveal two distinct features: the short-lived band, decaying along with the rise of the long living (on the time scale of the experiment) band at $\lambda=465 \mathrm{~nm}$. The latter is clearly the triplet-triplet absorption, very similar to the $T_{n} \leftarrow T_{1}$ absorption of the structurally closely related carbonyl compound, DMABA [20,21].

The absorption band close to $\lambda \approx 500 \mathrm{~nm}$ resembles very much that of the TICT state of DMAA (identified as characteristic of the species emitting the $F_{\text {a }}$ fluorescence) $[12,13]$, and is reproducing well the absorption of the radical ion acetophenone ${ }^{-}$[22] (Fig. 7) $)^{\ddagger}$.

Thus, it is to emphasize that the polar emitting state of DOAA is of very similar electronic structure to DMAA, characterized by the absorption localized in the radical anion $\left(\mathrm{A}^{-}\right) \pi$-electronic subsystem, evidently decoupled from the amino group. The only reasonable model for the decoupling is by the intramolecular $\approx 90^{\circ}$ rotation: it is a verification of the TICT model.

\subsection{Long-chain effects}

As to the effect of the long-chain alkyls in the amino group (in fact, the main effect appears between the $\mathrm{Me}_{2} \mathrm{~N}$ - and $\mathrm{Et}_{2} \mathrm{~N}$ - substituted compounds), the comparison of photophysics of DMAA and DOAA, as well as of DMABN and its long-chain homologues [6], can be stressed in the following.

$\$$ The difference between the spectra of DOAA and DMAA - absence of the triplet absorption in the older spectrum [10] - is due in part to different solvents, but mainly to the loss of sensitivity of the one-time photodiodes in the short-wa ve range, $\lambda<470 \mathrm{~nm}$. 


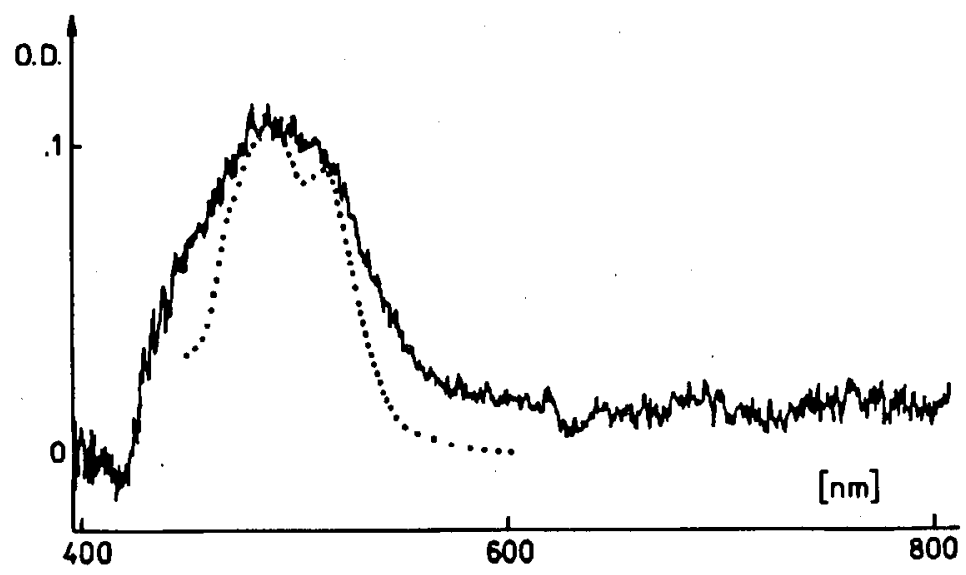

Fig. 7. Transient absorption spectrum of DOAA in ACN, $T=293 \mathrm{~K}, t_{\mathrm{d}}=100 \mathrm{ps}$. For comparison (dotted curve): the absorption spectrum of radical anion acetophenone ${ }^{-}$in methyltetrahydrofurane (MTHF), $T=77 \mathrm{~K}[22]$.

(i) The emission spectra of the compounds with larger alkyl substituents have a markedly higher $\phi_{A} / \phi_{B}$ ratio than the $-\mathrm{NMe}_{2}$ derivatives, the fluorescence $F_{\mathrm{a}}$ of the homologues of DMABN appearing even in hydrocarbon solvents;

(ii) The $F_{\mathrm{a}}$ transition is shifted with large alkyls to somewhat higher energies than that of the $-\mathrm{NMe}_{2}$ derivatives; this difference is increasing with the polarity of the solvent.

In all studied cases the two emitting states rapidly (as compared to the lifetime) approach the equilibrium, which does not necessarily mean the equilibrium is governing the fluorescence yield. Taking as the limit of reversibility the maximum at the plot $\ln \phi_{A} / \phi_{B}$ vs. $1 / T$ [23], we find that in ACN the critical temperature $T_{1}=294 \mathrm{~K}$ for DMAA [23], and $T_{1}=315 \mathrm{~K}$ for DOAA; i.e., in each case both, the equilibrium and the kinetics, play a non-negligible role. The radiative rates, $k_{f}$, seem not to change with the alkyl size [6].

Contrary to the $F_{\mathrm{a}}$ emission, the $F_{\mathrm{b}}$ band of DOAA is slightly shifted to lower energy as compared to DMAA (Table). To calculate the $h \nu_{\max }$ value of the $F_{\mathrm{a}}$ fluorescence of DOAA according to the published model [24], we used as the oxidation potential of trioctylamine that reported for tripentylamine in ACN, $E_{\text {ox }}=+0.89 \mathrm{~V}$ vs. $\mathrm{SCE}[25 \mathrm{a}]$, and the Onsager radius $\left(a_{0}^{3}=143 \AA^{3}\right)$ resulting from the molecular volume. Assuming the ground state potential barriers and Stokes' shifts in the $F_{\mathrm{b}}$ fluorescence to be the same for DOAA and DMAA, we obtain $19000 \mathrm{~cm}^{-1}$ as the calculated value, in a satisfactory agreement with the observed fluorescence maximum: $18600 \mathrm{~cm}^{-1}$ (Fig. 8).

Thus, it seems that the effect of large alkyl groups, increasing the intensity ratio of two fluorescence bands, $F_{\mathrm{a}} / F_{\mathrm{b}}$, is mainly due to the lowering of the oxidation potential, i.e. increasing the donor ability, of the dialkylamino group. $\$$

\$There are, however, substantial differences between the reported oxidation potentials of trialkylamines in $\mathrm{ACN}: \mathrm{NMe}_{3}+1.04 \mathrm{~V}[25 \mathrm{~b}] ; \mathrm{NEt}_{3}+1.15 \mathrm{~V}[25 \mathrm{c}] ; \mathrm{NProp}_{3}+0.88 ;+0.91 ;+0.93 \mathrm{~V}$ [25d]; +1.14 V [25f]; $\mathrm{NBu}_{3}+0.78 \mathrm{~V}$ [25e]; $\mathrm{NPent}_{3}+0.89 \mathrm{~V}$ [25a]. All values are given here vs. 


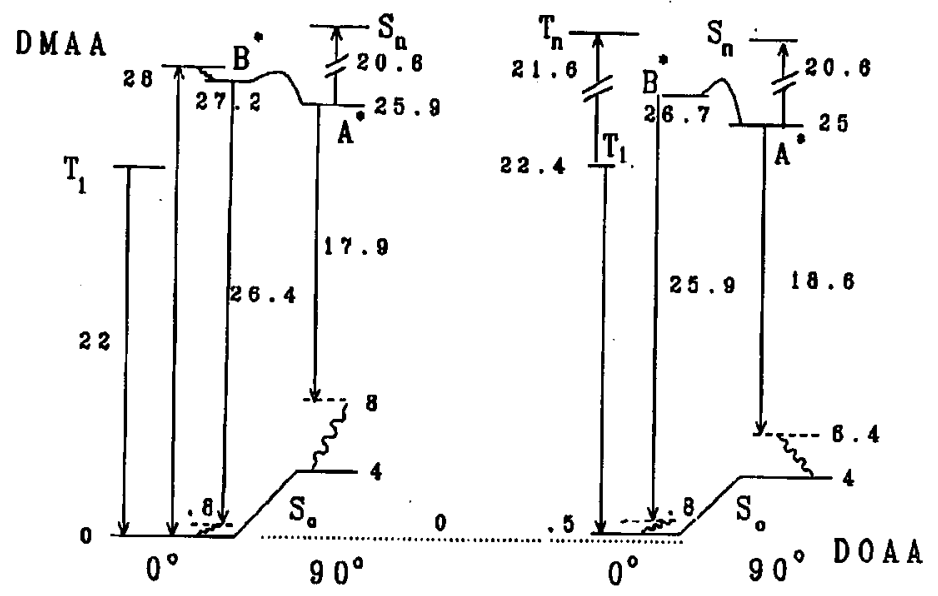

Fig. 8. Energy levels and transitions (in $10^{3} \mathrm{~cm}^{-1}$ ) of DMAA and DOAA (in ACN solution, unless otherwise stated). For DMAA the $(0,0)$ transition in phosphorescence is measured in MTHF; the $(0,0)$ transition in fluorescence is measured in $n$-propanol, $T=77 \mathrm{~K}[9]$; the Franck-Condon relaxation in liquid ACN is equally divided into the excited and ground state contributions. The same Franck-Condon relaxation is assumed for $F_{\mathrm{b}}$ emission in DOAA. The height of the ground state potential barrier is estimated as $0.5 \mathrm{eV}$, the ground state relaxation after the $F_{\mathrm{a}}$ emission (destabilization energy) is calculated according to the previous model [24]. Setting the ground state barrier height at a lower level (as, e.g. in Ref. [30]) would not change the comparison of DOAA and DMAA, except the energy difference between the $B^{*}$ and $A^{*}$ states.

Marginally, it is to admit that the entropy factor, $T \Delta S_{0}$, is neglected in the model calculation [24]; analyzing the scarce reported data on the solubility of tetraalkylammonium salts [28] we find that the solvation entropy of the $\mathrm{NR}^{+}$ ions in aprotic solvents is sometimes considerably increasing with the length of the alkyl chain. Entropy effects in different solvents, in dependence on the length of the alkyl chains, seem to be worth attention.

\subsection{Inversional mode?}

In their very stimulating papers $[6,7,29]$ Zachariasse, Warman et al. suppose that the main factor determining the dual fluorescence ability in DMABN and in related compounds is the proximity of two close-laying ${ }^{1} \pi, \pi^{*}$ states. In all compounds of the studied structural families which exhibit the dual fluorescence, there are, indeed, two neighbouring electronic states (or even three, as in DMAA

the saturated calomel electrode (SCE). As these are irreversible potentials, they strongly depend on the experimental conditions of the measurement. The effect can also be due to steric interactions of higher alkyls with the aromatic ring, resulting in non-planarity in the ground state. The effect of replacement of Me by Et was observed and discussed in the context of the TICT states of dialkylamino-pyrimidines [26]. The deviations from coplanarity (non-zero initial twist angle) should primarily influence the kinetics of the process. Now, it seems rather that the reported irreversible electrochemical oxidation potential of $\mathrm{NEt}_{3}$ may be wrong, specially as the vertical ionization potential decreases at the substitution of methyls by ethyl groups [26, 27]. 
where the ${ }^{1} n, \pi^{*}$ state plays an important role in less polar solvents [9]). The cited Authors [6, 7, 29] assume, however, the molecule is non-planar because of a pyramidalization of the $N$ atom of the dialkylamino group; the inversional mode is assumed to couple the two states which results (in an unexplained way) in formation of the polar form, without mutual twisting of $D$ and $A$.

Very recently, two important theoretical papers appeared, both with similar conclusions in this respect. Roos et al. [8] demonstrated that the pyramidalization of DMABN does not lead to any markedly polar structures. Very similar results are obtained by Gorse and Pesquer [30] for both, DMABN and the benzaldehyde derivative DMABA, including the solvation effects calculated for strongly polar solvents. But even a simple qualitative inspection of the symmetry rules for vibronic coupling [31] compels us to reject the concept of inversion.

In the planar DMABN ( $C_{2 v}$ symmetry) the two states involved are the lowest excited ${ }^{1} B_{1}$ and the next ${ }^{1} A_{1}$ state. If we assume a pyramidalization of the amino nitrogen (without internal rotation), the point symmetry is reduced to $C_{s}$, with the plane of symmetry perpendicular to the aromatic ring. The symmetry of the two excited states turns to be: $A_{1} \rightarrow A^{\prime}, B_{1} \rightarrow A^{\prime \prime}$. The inversional mode is symmetric in the $C_{s}$ point group, $a^{\prime}$; it cannot couple the $A^{\prime}$ and $A^{\prime \prime}$ states.

Thus, one can eliminate the hypothesis of the inversional coupling, not excluding a role of the proximity effects in the dynamics of the process.

\subsection{The nature of the highly polar slate}

Summarising the results on the photophysics of DOAA, we can conclude:

(i) There is an excellent similarity between the spectra and behaviour of DOAA and DMAA or other $p$-carbonyl substituted dimethylanilines which have a rotable (not immobilized) $-\mathrm{NR}_{2}$ group $[9,10,21,12]$.

(ii) Transient absorption spectra and the fluorescence spectra (energy of transition) give evidence that in the highly polar emitting state the separation of charges is connected with the decoupling of $D^{+}\left(-\mathrm{NR}_{2}^{+}\right)$and $A^{-}$(acetophenone radical anion) structural subunits of the molecule.

(iii) These are just the main features of the TICT state. The TICT model of the structural change is also supported by all quantum chemical calculations, e.g. $[8,9,30]$.

\section{Acknowledgments}

The authors are indebted to Dr Claude Rullière for stimulating cooperation before starting the present work; to Dr Klaas Zachariasse for inspiring discussions; to Dr Andrei Mikhailov (EKSPLA, Vilnius) and to Dr Audrius Pugžlys (Vilnius University) for their help in solving numerous apparative problems; and to Andrzej Ardasiewicz Co. (Warsaw) for designing the electronic detection system operating the photodiode arrays. The work was supported in part by the State Committee for Scientific Research (Republic of Poland), grant \# 2365-91-01. 


\section{References}

[1] E. Lippert, W. Lüder, H. Boos, in: Advances in Molecular Spectroscopy, Ed. A. Mangini, Pergamon Press, Oxford 1962, p. 443.

[2] W. Rettig, Topics in Current Chem. 169, 253 (1994).

[3] Z.R. Grabowski, K. Rotkiewicz, A. Siemiarczuk, D.J. Cowley, W. Baumann, Nouv. J. Chim. 3, 443 (1979).

[4] Z.R. Grabowski, Pure Appl. Chem. 64, 1249 (1992); 65, 1751 (1993).

[5] M.C.C de Lange, D. Thorn Leeson, K.A.B. van Kuijk, A.H. Huizer, C.A.G.O. Varma, Chem. Phys. 174, 425 (1993); ibid. 177, 243 (1993).

[6] W. Schuddeboom, S.A. Jonker, J.M. Warman, U. Leinhos, W. Kühnle, K.A. Zachariasse, J. Phys. Chem. 96, 10809 (1992).

[7] K.A. Zachariasse, T. von der Haar, A. Hebecker, U. Leinhos, W. Kühnle, Pure Appl. Chem. 65, 1745 (1993).

[8] L. Serrano-Andrés, M. Merchán, B.O. Roos, R. Lindh, J. Am. Chem. Soc. 117, 3189 (1995).

[9] J. Dobkowski, E. Kirkor-Kamińska, J. Koput, A. Siemiarczuk, J. Lumin. 27, 339 (1982).

[10] Z.R. Grabowski, J. Dobkowski, W. Kühnle, J. Molec. Structure 114, 93 (1984).

[11] T. Okada, N. Mataga, W. Baumann, J. Phys. Chem. 91, 760 (1987).

[12] C. Rullière, Z.R. Grabowski, J. Dobkowski, Chem. Phys. Lett. 137, 408 (1987).

[13] Z.R. Grabowski, in: Supramolecular Photochemistry, Ed. V. Balzani, Reidel, Dordrecht 1987, p. 319.

[14] A. Déclémy, C. Rullière, Rev. Sci. Instrum. 57, 2733 (1986).

[15] J. Jasny, J. Lumin. 17, 149 (1978).

[16] C. Rullière, A. Déclémy, Ph. Pée, Rev. Phys. Appl. 18, 347 (1983).

[17] J. Jasny, J. Sepioł, J. Karpiuk, J. Gilewski, Rev. Sci. Instr. 65, 3646 (1994).

[18] E. Kirkor-Kaminiska, K. Rotkiewicz, A. Grabowska, Chem. Phys. Lett. 58, 379 (1978).

[19] J. Dobkowski, J. Waluk, W. Yang, C. Rullière, W. Rettig, to be published.

[20] D.J. Cowley, I. Pasha, J. Chem. Soc., Perkin Trans. II, 1139 (1983).

[21] J. Dobkowski, Z.R. Grabowski, E. Heumann, D. Khechinashvili, B. Kozankiewicz, W. Kühnle, J. Sepioł, in: Inlernat. Symp. on Molecular Luminescence and Photophysics - Half a Century of the Jabłoniski Diagram, Inst. of Physics, N. Copernicus Univ., Torun 1986, p. 81.

[22] T. Shida, Electronic Absorption Spectra of Radical Ions, Elsevier, Amsterdam 1988, p. 275.

[23] Z.R. Grabowski, K. Rotkiewicz, W. Rubaszewska, E. Kirkor-Kamińska, Acta Phys. Pol. A 54, 767 (1978).

[24] Z.R. Grabowski, J. Dobkowski, Pure Appl. Chem. 55, 245 (1983).

[25] L. Meites, P. Zuman, CRC Handbook Series in Organic Electrochemistry, Vol. 1, CRC Publ. Co., Cleveland, (a) p. 666; (b) p. 64; (c) p. 252; (d) p. 396; (e) p. 550; (f) ibid. Vol. 3, p. 148.

[26] J. Herbich, Z.R. Grabowski, H. Wójtowicz, K. Golankiewicz, J. Phys. Chem. 93, 3439 (1989). 
[27] D.H. Aue, H.M. Webb, M.T. Bowers, J. Am. Chem. Soc. 98, 311 (1976).

[28] P.T. Walden, Z. Phys. Chem. 55, 698, 712 (1906).

[29] K.A. Zachariasse, Th. von der Haar, A. Hebecker, U. Leihos, W. Kühnle, in: XVIth Internat. Conf. on Photochemistry, Abstracts, Univ. of British Columbia, Vancouver 1993 , p. 83.

[30] A.D. Gorse, M. Pesquer, J. Phys. Chem. 99, 4039 (1995).

[31] R.M. Hochstrasser, C. Marzacco, J. Chem. Phys. 49, 971 (1968). 